\title{
Two genetic variants in telomerase-associated protein 1 are associated with stomach cancer risk
}

\author{
Dong-Hao Jin ${ }^{1}$, Sung Kim², Duk-Hwan Kim ${ }^{1}$ and Joobae Park ${ }^{1}$
}

This study examined the impact of two single-nucleotide polymorphisms (SNPs) in the telomerase-associated protein 1 (TEP1) gene on the risk of breast, colorectal, hepatocellular, lung and stomach cancer. A significantly increased stomach cancer risk associated with the GG genotype at rs 1760893 (odds ratio $(O R)=1.64,95 \%$ confidence interval $(\mathrm{CI})=1.23-2.20, P=0.004$ ) or CC genotype at rs 1713423 (OR $=2.40,95 \% \mathrm{Cl}=1.88-3.07, P<0.0001$ ) was observed, compared with their wild-type counterpart. The GG genotype at rs 1760893 was also associated with enhanced hepatocellular cancer susceptibility $(0 R=1.46$, $95 \% \mathrm{Cl}=1.05-2.03, P=0.02$ ). In classification and regression tree analysis, individuals carrying the $\mathrm{CC}$ genotype at rs 1713423 had 2.69 -fold increased risk of stomach cancer $(95 \% \mathrm{Cl}=2.18-3.32, P<0.0001)$ compared with the TT and TC genotypes. The current results suggested that genetic variants at TEP1 SNPs rs1760893 and rs1713423 may be associated significantly with increased risk of stomach cancer.

Journal of Human Genetics (2016) 61, 885-889; doi:10.1038/jhg.2016.71; published online 16 June 2016

\section{INTRODUCTION}

Cancer is the leading cause of death worldwide. Lung, liver, stomach, colorectal and breast cancers cause the most cancer deaths each year. ${ }^{1}$ Inherited genetic is one determinable factor of cancer causes, and the most common type of inherited genetic variant is the single-nucleotide polymorphism (SNP). SNP is a DNA sequence variation occurring within a population in which a single nucleotide in the genome differs between members of a biological species or paired chromosomes. Most commonly, SNPs are found in the DNA between genes, but when SNPs occur within a gene or in a regulatory region near a gene, they may have a role in disease by affecting the gene's function, resulting in an increased disease susceptibility or protection from disease. ${ }^{2}$

Telomerase-associated protein 1 (TEP1) is a component of the telomerase ribonucleoprotein complex and is thought to responsible for catalyzing the addition of new telomeres to chromosomes. ${ }^{3}$ The human TEP1 gene resides on chromosome 14q11.2, and there are two isoforms of this protein, the isoforml is 2627aa and the isoform 2 is 2519 aa. The isoform 2 lacks exons 5 and 6 compared with the isoform $1 .{ }^{4}$ The mRNA expression of TEP1 is increased in tumor samples compared with normal breast tissue..$^{5}$ TEP1 protein is more abundant in higher-grade ovarian cancer. ${ }^{6}$ TEP1 is also a component of the vault particle, a large cytoplasmic ribonucleoprotein complex consisting of major vault proteins, vault poly polymerase, small vault RNAs and TEP1. ${ }^{7}$ TEP1 protein is required for vault RNA stability and its association with the vault particle. ${ }^{8}$ Vaults are associated with the nuclear pore complex and have a role in the transportation of molecules, such as mRNA, from the nucleus to parts of the cytoplasm. ${ }^{9}$ Vaults are overexpressed in cancer patients diagnosed with multidrug resistance and have been related with the resistance against many chemotherapy treatments. ${ }^{10-12}$

Several studies have analyzed the association of genetic variants in TEP1 with the susceptibility to certain cancers. For example, Chang et al. ${ }^{13}$ revealed the significant correlation of four SNPs (rs2228041, rs2228026, rs1713418 and rs2297615) in the TEP1 gene with the increased risk of bladder cancer. Andrew et al. ${ }^{14}$ observed that another TEP1 SNP (rs1760897) is associated with enhanced bladder cancer susceptibility. In addition, Jung et al. ${ }^{15}$ studied the impact of telomere maintenance gene polymorphisms on hepatocellular carcinoma and reported that the SNP at rs1713449 in TEP1 is strongly associated with increased risk of hepatitis B virus-associated hepatocellular cancer development.

Recently, we carried out a genome-wide association study between SNPs and risk of stomach cancer in Korean population ${ }^{16}$ and identified that two SNPs in the TEP1 gene (rs1760893, rs1713423) were correlated with high risk of this cancer. In this study, to further analyze haplotypes, to increase statistical power and to determine whether the two SNPs was also involved in other cancers, we compared the prevalence of genotypes at these two SNPs between a group of 3065 patients who had 5 different types of cancers (breast, colorectal, hepatocellular, lung, stomach) and a control group of 1375 individuals.

\section{MATERIALS AND METHODS}

Study populations

The population consisted of 1375 controls and 590 patients with breast cancer, 678 patients with colorectal cancer, 598 patients with hepatocellular carcinoma,

\footnotetext{
${ }^{1}$ Department of Molecular Cell Biology, Samsung Biomedical Research Institute, Sungkyunkwan University School of Medicine, Suwon, Korea and ${ }^{2}$ Department of Surgery, Samsung Medical Center, Sungkyunkwan University School of Medicine, Seoul, Korea

Correspondence: Professor DH Kim or Professor J Park, Department of Molecular Cell Biology, Samsung Biomedical Research Institute, Sungkyunkwan University School of Medicine, \#50 IIwon-dong, Kangnam-Ku, Room B155, Seoul 135-710, Korea.

E-mail:dukhwan.kim@samsung.com or jb.park@skku.edu

Received 13 January 2016; revised 26 April 2016; accepted 30 April 2016; published online 16 June 2016
} 
299 patients with lung cancer and 900 patients with gastric cancer from the general population. They were used for the calculation of allele frequencies of the SNPs and for the construction of haplotypes. All cancer patients underwent curative surgical resection at the Samsung Medical Center, a major referral center for cancer in Seoul, Korea from 2003 to 2012. ${ }^{2}$ Age- and sex-matched control participants for the five cancers were selected from among healthy individuals who visited the Samsung Medical Center for regular health checkups. Peripheral blood samples from all participants were collected according to ethical guidelines on the use of human tissues and biological samples in research after obtaining appropriate institutional review board permission and written informed consent from all participants. Information regarding demographics was obtained by using an intervieweradministered questionnaire.

\section{Genotyping}

Genomic DNA from peripheral blood lymphocytes was extracted using a QIAamp Blood Kit (Qiagen, Valencia, CA, USA). DNA quality was checked using an ultraviolet (UV) spectrophotometer (Pharmacia Biotech, Cambridge, UK) and the PicoGreen Double-Stranded DNA Quantitation Kit (Molecular Probes, Eugene, OR, USA) using a SpectraMax Gemini UV spectrometer (Molecular Devices, Sunnyvale, CA, USA). Genotyping was performed using the homogeneous MassExtend assay (Sequenom, San Diego, CA, USA), as described previously. ${ }^{17}$ Both the amplification and extension primers were designed using the Sequenom Assay Design program. PCR and primer extension reactions were performed according to the protocol for hME procedure (MassARRAY System Training User's Guide; Sequenom).

\section{Classification and regression tree (CART) analysis}

CART analysis of the two SNPs (rs1760893, rs1713423) was performed using the stomach cancer and control samples. ${ }^{18-20}$ The initial split was by rs 1713423 and the second split was by rs1760893. Each node contains frequencies and percentages of case and controls. Subgroups of individuals with differential risk associations with stomach cancer were identified in the different terminal nodes of the tree. Odds ratios (ORs), 95\% confidence intervals (CIs) and $P$-values were computed.

Table 1 Demographic distribution

\begin{tabular}{lcccc}
\hline & Age in years, mean \pm s.d. & Total & Men & Women \\
\hline Control & $60 \pm 14$ & 1375 & 1072 & 303 \\
& & & & \\
Cancer & & & & 590 \\
Breast & $49 \pm 10$ & 590 & & 118 \\
Colorectal & $60 \pm 11$ & 678 & 560 & 118 \\
Hepatocellular & $53 \pm 10$ & 598 & 480 & 73 \\
Lung & $59 \pm 11$ & 299 & 226 & 274 \\
Stomach & $57 \pm 12$ & 900 & 626 & \\
\hline
\end{tabular}

*Breast cancer is female-matched.

\section{Statistical analyses}

The association between genotypes and the prevalence of each cancer was analyzed using Pearson chi-square test and Cochran-Armitage test for trend. Multivariate logistic regression analysis was performed using a web tool for SNP analysis (SNPStats, Barcelona, Spain) in both the patient and control groups. ${ }^{21}$ ORs, 95\% CIs and $P$-values were obtained. All $P$-values were twotailed and a level $<0.05$ was considered statistically significant.

\section{RESULTS \\ Subject characteristics}

The study population comprised of 3065 cancer patients (breast cancer, $n=590$; colon cancer, $n=678$; hepatocellular cancer, $n=598$; lung cancer, $n=299$; and stomach cancer $n=900)$ and 1375 healthy individuals (Table 1). Men accounted for about three times the number of women in both the control and patient groups. The mean age ( \pm s.d.) was $49 \pm 10$ years in breast cancer, $60 \pm 11$ years in colorectal cancer, $53 \pm 10$ years in hepatocellular cancer, $59 \pm 11$ years in lung cancer, $57 \pm 12$ years in stomach cancer and $60 \pm 14$ years in the control group. The human TEP1 gene is located on chromosome 14q11.2 from base 20365667 to 20414172 in GRCh38 and has 11 transcript splice variants. The longest transcript length is 10694 bps and there are 54 exons (Figure 1). The rs1760893 SNP was identified in the promoter region and the rs1713423 in the twelfth intron of the TEP1 gene.

Genotypic distribution of the two SNPs in the five human cancers Genotypic distributions at the two SNPs in the five cancer types were compared with the control group (Table 2). Genotypic distributions at rs1760893 and rs1713423 in breast, colorectal, hepatocellular and lung cancer were similar to that in the control group. But in patients with stomach cancer, the distribution differed significantly. The frequencies of the GG genotype at rs1760893 and the CC genotype at rs1713423 were significantly higher in the stomach cancer group than in the control group (rs1760893: $15 \%$ vs $10 \%$, Chi-square $P=0.003$; rs1713423: $35 \%$ vs 19\%, Chi-square $P<0.0001$ ).

The linkage disequilibrium structure of TEP1 SNPs in the populations of Han Chinese in Beijing and Japanese in Tokyo (CHB $+\mathrm{JPT}$ ) was analyzed by Haploview ${ }^{22}$ (Supplementary Figure S1). No strong combination between rs1760893 and rs1713423 was observed in CHB+JPT ( $D^{\prime}$-value: 0.15$)$ and our Korean $\left(D^{\prime}\right.$-value: $0.20)$ populations. The haplotype distribution was similar between the Korean (control group) and CHB+JPT populations (Supplementary Table S1). However, haplotypes of CT and CG had 1.42-times (95\% $\mathrm{CI}=1.18-1.71 ; \quad P=0.0002)$ and 1.81-times $(95 \% \mathrm{CI}=1.52-2.15$; $P<0.0001)$ enhanced risk of stomach cancer compared with the haplotype TT.

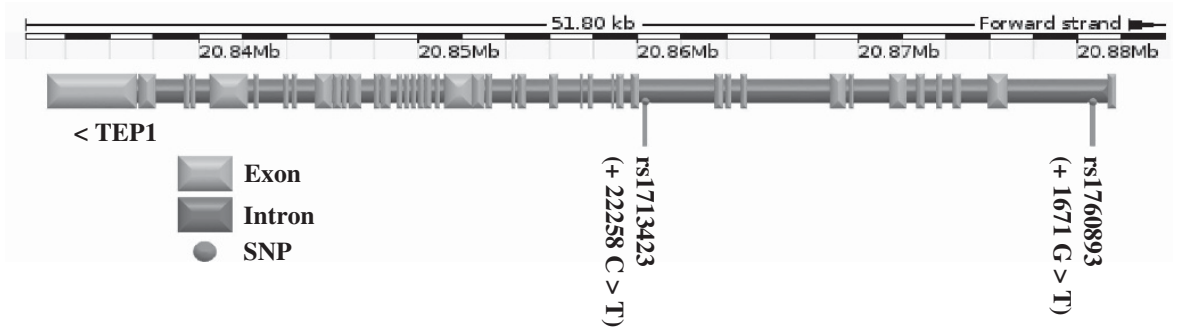

Figure 1 Location of the two single-nucleotide polymorphisms (SNPS) in the TEP1 gene is illustrated. The first base of the transcription start site is counted as position +1 (GenBank accession no. NC-000014.9). $\mathrm{G} \rightarrow \mathrm{T}$ indicates guanine to thymine, and $\mathrm{C} \rightarrow \mathrm{T}$ indicates cytosine to thymine. A full color version of this figure is available at the Journal of Human Genetics journal online. 
Table 2 Association of individual SNP with the prevalence of cancers

Cancers

\begin{tabular}{|c|c|c|c|c|c|c|}
\hline & & & & & & \\
\hline Genotype & Control (\%) & Breast (\%) & Colorectal (\%) & Hepatocellular (\%) & Lung (\%) & Stomach (\%) \\
\hline$S N P_{-} 1$ (rs 1760 & & & & & & \\
\hline $\mathrm{GG}$ & $120(10)$ & $53(10)$ & $69(9)$ & $76(14)$ & $29(10)$ & $119(15)$ \\
\hline GT & $512(45)$ & $220(42)$ & $385(49)$ & $238(44)$ & $133(47)$ & $353(45)$ \\
\hline TT & $517(45)$ & $248(48)$ & $338(43)$ & $224(42)$ & $123(43)$ & $312(40)$ \\
\hline $\begin{array}{l}\text { Total } \\
p^{a}\end{array}$ & 1149 & 521 & 792 & 538 & 285 & 784 \\
\hline Chi-square $^{b}$ & & 0.61 & 0.16 & 0.08 & 0.81 & 0.003 \\
\hline Trend $^{c}$ & & 0.41 & 0.84 & 0.04 & 0.72 & 0.002 \\
\hline SNP_2(rs1713 & & & & & & \\
\hline $\mathrm{CC}$ & 210 (19) & $40(15)$ & $158(21)$ & $113(22)$ & $8(14)$ & $289(35)$ \\
\hline $\mathrm{TC}$ & $539(48)$ & $139(51)$ & $321(44)$ & $245(48)$ & $34(60)$ & 322 (39) \\
\hline $\mathrm{TT}$ & $372(33)$ & $92(34)$ & $256(35)$ & $153(30)$ & $15(26)$ & $213(26)$ \\
\hline $\begin{array}{l}\text { Total } \\
P^{a}\end{array}$ & 1121 & 271 & 735 & 511 & 57 & 824 \\
\hline Chi-square $^{b}$ & & 0.30 & 0.14 & 0.20 & 0.23 & $<0.0001$ \\
\hline Trend $^{c}$ & & 0.32 & 0.74 & 0.08 & 0.82 & $<0.0001$ \\
\hline
\end{tabular}

Abbreviation: SNP, single-nucleotide polymorphism

a $P$-values were based on the association between control and each cancer.

bearson chi-square test.

cCochran-Armitage trend test.

\section{Multivariate logistic regression analysis}

Multivariate logistic regression analysis was performed to determine the effect of genotypic variants at rs1760893 and rs1713423 on the risk of the five cancers (Table 3). Genotypic variants at these two SNPs were not associated significantly with susceptibility of breast, colorectal and lung cancers. However, individuals who had the GG genotype at rs1760893 had a 1.46-times greater risk of hepatocellular cancer (codominant model, 95\% CI $=1.05-2.03 ; P=0.02$ ) and a 1.64-times greater risk of stomach cancer. In addition, the CC genotype at rs1713423 was associated with a significantly increased risk of stomach cancer (codominant model, $\mathrm{OR}=2.40 ; \quad 95 \% \quad \mathrm{CI}=1.88-3.07$; $P<0.0001)$ compared with the TT genotype. The enhanced susceptibility was also observed in the dominant and recessive models (Supplementary Table S2).

\section{CART analysis of the rs1760893 and rs1713423 SNPs in stomach cancer}

The rs1760893 and rs1713423 SNPs that had been significantly associated with stomach cancer risk were further analyzed for their interaction in the CART analysis (Figure 2). The initial split was by rs1713423 because genetic variant at this SNP represented higher susceptibility than the SNP at rs1760893 (OR: 2.40 vs 1.64). Individuals who had the TT or TC genotype at rs1713423 represented $33.8 \%$ of the stomach cancers. However, individuals with the CC genotype at this region constituted $57.9 \%$ of the stomach cancers. Further split of individuals with the CC genotype at rs1713423 (Node2) into binary subgroups by rs1760893 determined that the percentage of patients with the TT or TG genotype at rs1760893 was $58 \%$ (Node3) and the percentage of patients with the GG genotype was $57.3 \%$ (Node4). There was no significant increase of patient proportion from node 2 to node 3 or node4. Table 4 summarizes the risk estimates for individuals in each node. Individuals who had the variant homozygote (CC genotype) at rs1713423 had a 2.69-times enhanced risk of stomach cancer $(95 \% \mathrm{CI}=2.18-3.32 ; P<0.0001)$ compared with individuals with the wild-type homozygote or heterozygote (TT or TC genotype). In addition, individuals who had a variant homozygote (CC genotype) at rs1713423 and a variant homozygote (GG genotype) at rs1760893 did not have a further increased risk of stomach cancer (OR: 2.69 vs 2.63), indicating no cumulative effect of the two SNPs on stomach cancer susceptibility.

\section{Impact of genetic variant at rs1760893 or rs1713423 on TEP1 expression}

The impact of genetic variant at rs1760893 or rs1713423 on TEP1 expression was analyzed by using a database portal The Genotype-Tissue Expression (GTEx) (http://gtexportal.org). ${ }^{23,24}$ However, there was no data available for SNPs rs1760893 and rs1713423 in this database. We then determined SNPs that were near the rs1760893 or rs1713423 and correlated significantly with TEP1 expression in this database (Supplementary Figure S2A). In result, seven SNPs rs1760891, rs1713434, rs4246977, rs1713435, rs1760889, rs1760955 and rs1713428 near the rs1760893 were identified to associate significantly with the gene's expression. Linkage disequilibrium structure analysis revealed that rs1760893 has a strong recombination with the other SNPs in the populations of $\mathrm{CHB}+\mathrm{JPT}$ and CEU+TSI (Northern and Western European and Toscans in Italy) (Supplementary Figures S2B and C). These results suggested that genetic variant at rs1760893 may be associated with the TEP1 expression.

\section{DISCUSSION}

In this study, the association of genetic variants at two SNPs (rs1760893, rs1713423) in the TEP1 gene with breast, colorectal, hepatocellular, lung and stomach cancer risk was investigated. The rs1760893 and rs1713423 SNPs correlated significantly with an increased risk of stomach and/or hepatocellular cancer, suggesting that genetic variants in these two regions may affect stomach and/or hepatocellular cancer susceptibility. SNPs occurring within a gene or 
Table 3 Multivariate logistic regression analysis of individual SNP and cancer risk

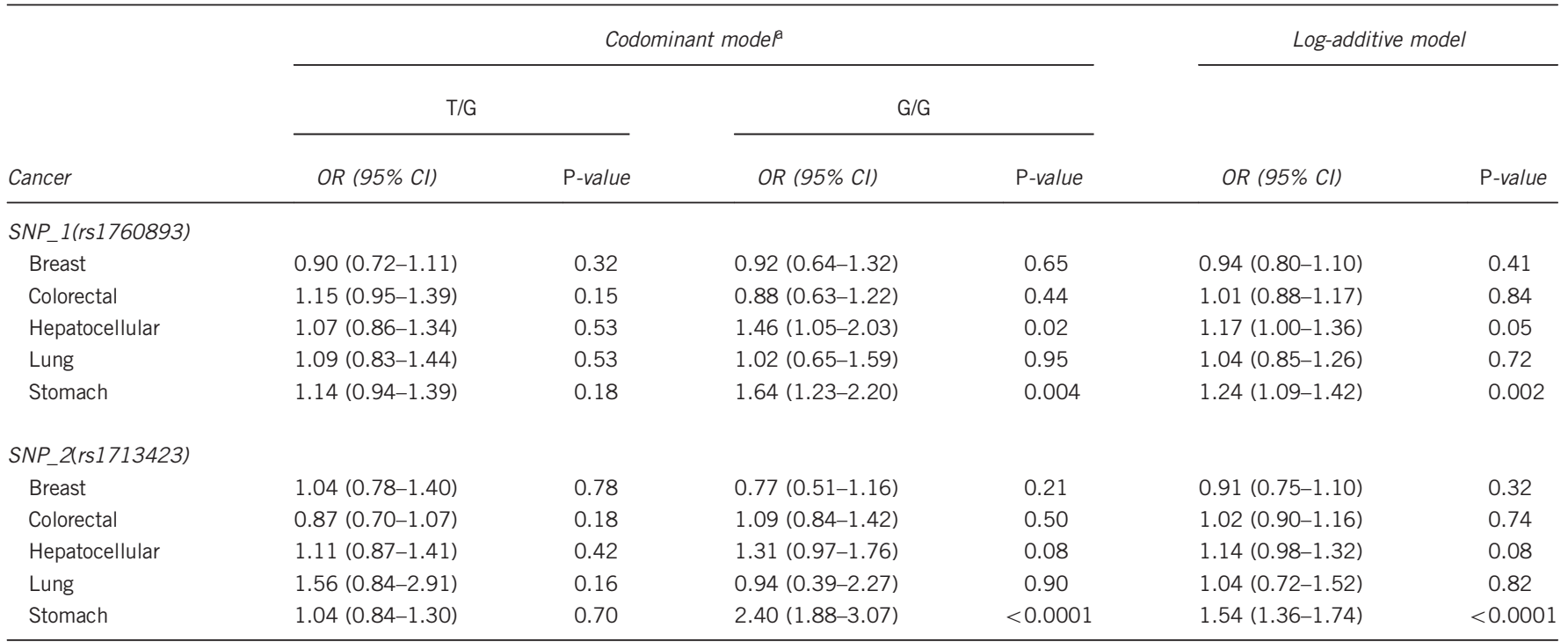

Abbreviations: $\mathrm{Cl}$, confident interval; OR, odds ratio; SNP, single-nucleotide polymorphism.

aTT was the reference in the codominant model.

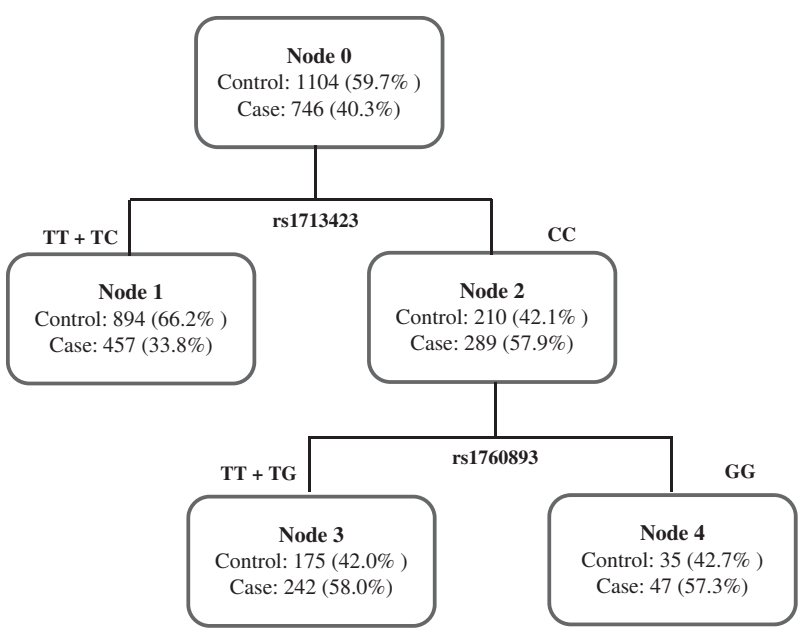

Figure 2 Tree structure of the classification and regression tree (CART) analysis of the two SNPs (rs1760893, rs1713423) in stomach cancer. The initial split was by rs1713423 and the second split was by rs1760893. Each node contains frequencies and the percentages of case and controls.

in a regulatory region near a gene may have a role in disease by affecting the gene's function. As rs1760893 is located at the promoter region of TEP1, this variation may regulate the gene's expression by altering transcriptional efficacy. Substantial differences in the transcriptional level result from genetic variants in the promoter regions of genes. $^{25,26}$ The rs1713423 SNP, which is located within the intron of TEP1, may affect splicing of the TEP1 transcript or disturb its expression by altering transcription factor-binding sites.

Although telomerase activity is associated with TEP1, this protein is not necessary for proper telomerase function and is not a component of the core telomerase complex. ${ }^{27}$ Use of TEP1-deficient mice established that TEP1 is not essential for telomerase activity or telomere length maintenance in vivo. ${ }^{28}$ However, TEP1, heat-shock protein 90 and topoisomerase II $\alpha$ associate directly with bloom syndrome protein (BLM) helicase in immortalized cells using the alternative lengthening of telomere (ALT) pathway and modulate its helicase activity. ${ }^{29}$ Cells deficient in BLM helicase display increased telomere association between homologous chromosomes. BLM helicase is important for proper telomere maintenance and function in ALT cells. ${ }^{30}$ TEP1 can have a role in telomere maintenance in ALT cells. About $5-10 \%$ of human cancers rely on the ALT pathway to maintain telomere length. ${ }^{31}$

The expression of TEP1 is prevalent in multiple human tissues, including the lung, liver and stomach. In one study, TEP1 was expressed in $93 \%$ of 92 human lung cancer tissue samples and all 92 corresponding non-neoplastic lung tissues. ${ }^{32}$ TEP1 was also reportedly expressed in all 23 human hepatocellular carcinoma samples and corresponding adjacent liver tissue. ${ }^{33}$ In other studies, the positive rate of TEP1 mRNA from 24 human gastric cancer samples and corresponding non-cancerous tissues was $100 \%$ and $91.7 \%$, respectively. ${ }^{34}$ There was no significant difference of TEP1 expression between cancer and corresponding non-cancerous tissues in these three tissue types. TEP1 is known to exert its role through an interaction with molecules, such as major vault proteins, ${ }^{7}$ small vault RNAs $^{7}$ and BLM. ${ }^{29}$ Therefore, their expression levels are quite important for TEP1 function and may be associated with TEP1-related cancer risk. Expression levels of major vault proteins were high in the stomach and colon, medium in the lung and liver and low in the breast. ${ }^{35}$ In contrast, BLM was high in the stomach, colon, lung and breast, but low in the liver. ${ }^{35}$ Accordingly, it is likely that different SNPs in TEP1 may confer different susceptibility to cancer development according to the expression levels of the proteins in each tissue.

In conclusion, TEP1 genetic variants at rs1760893 and rs1713423 were presently associated significantly with stomach cancer susceptibility. We also speculate that the genetic variant at rs1760893 located at TEP1 promoter region may influence the gene's expression. 
Table 4 Stomach cancer risk estimates of CART terminal nodes

\begin{tabular}{|c|c|c|c|c|c|}
\hline Node & Genotype & Control (\%) & Case (\%) & OR $(95 \% \mathrm{Cl})$ & P-value \\
\hline 2 & rs1713423 (V) & $210(42.1 \%)$ & $289(57.9 \%)$ & $2.69(2.18-3.32)$ & $<0.0001$ \\
\hline 4 & rs1713423(V)-rs1760893 (V) & $35(42.7 \%)$ & $47(57.3 \%)$ & $2.63(1.67-4.13)$ & $<0.0001$ \\
\hline
\end{tabular}

Abbreviations: CART, classification and regression tree; $\mathrm{Cl}$, confidence interval; $\mathrm{H}$, heterozygote; OR, odds ratio; $\mathrm{V}$, variant homozygote; W, wild-type homozygote.

\section{CONFLICT OF INTEREST}

The authors declare no conflict of interest.

\section{ACKNOWLEDGEMENTS}

This work was supported by grants from the National R\&D programs for Cancer Control, Ministry of Health \& Welfare (1520150) and from the Korea Health Industry Development Institute (KHIDI), funded by the Ministry of Health \& Welfare (HI14C1979), Republic of Korea.

1 WHO. All Cancers (Excluding Non-Melanoma Skin Cancer) Estimated Incidence, Mortality and Prevalence Worldwide in 2012. http://globocan.iarc.fr/Pages/fact sheets cancer.aspx (2012).

2 Kim, D. H., Park, S. E., Kim, M., Ji, Y. I., Kang, M. Y., Jung, E. H. et al. A functional single nucleotide polymorphism at the promoter region of cyclin A2 is associated with increased risk of colon, liver, and lung cancers. Cancer 117, 4080-4091 (2011).

3 Saito, T., Matsuda, Y., Suzuki, T., Hayashi, A., Yuan, X., Saito, M. et al. Comparative gene mapping of the human and mouse TEP1 genes, which encode one protein component of telomerases. Genomics 46, 46-50 (1997)

4 Harrington, L., McPhail, T., Mar, V., Zhou, W., Oulton, R., Bass, M. B. et al. A mammalian telomerase-associated protein. Science 275, 973-977 (1997).

5 Salhab, M., Jiang, W. G., Newbold, R. F. \& Mokbel, K. The expression of gene transcripts of telomere-associated genes in human breast cancer: correlation with clinico-pathological parameters and clinical outcome. Breast Cancer Res. Treat. 109, 35-46 (2008).

6 Szaflarski, W. Sujka-Kordowska, P., Pula, B., Jaszczynska-Nowinka, K., Andrzejewska, M., Zawierucha, P. et al. Expression profiles of vault components MVP, TEP1 and VPARP and their correlation to other multidrug resistance proteins in ovarian cancer. Int. J. Oncol. 43, 513-520 (2013).

7 Kickhoefer, V. A., Stephen, A. G., Harrington, L., Robinson, M. O. \& Rome, L. H. Vaults and telomerase share a common subunit, TEP1. J. Biol. Chem. 274 32712-32717 (1999)

8 Kickhoefer, V. A., Liu, Y., Kong, L. B., Snow, B. E., Stewart, P. L., Harrington, L. et al. The telomerase/vault-associated protein TEP1 is required for vault RNA stability and its association with the vault particle. J. Cell Biol. 152, 157-164 (2001).

9 Chugani, D. C., Rome, L. H. \& Kedersha, N. L. Evidence that vault ribonucleoprotein particles localize to the nuclear pore complex. J. Cell Sci. 106 (Pt 1), 23-29 (1993).

10 Mossink, M. H., van Zon, A., Scheper, R. J., Sonneveld, P. \& Wiemer, E. A. Vaults: a ribonucleoprotein particle involved in drug resistance? Oncogene 22 7458-7467 (2003).

11 Kickhoefer, V. A., Vasu, S. K. \& Rome, L. H. Vaults are the answer, what is the question? Trends Cell. Biol. 6, 174-178 (1996).

12 Kong, L. B., Siva, A. C., Rome, L. H. \& Stewart, P. L. Structure of the vault, a ubiquitous celular component. Structure 7, 371-379 (1999).

13 Chang, J., Dinney, C. P., Huang, M., Wu, X. \& Gu, J. Genetic variants in telomere-maintenance genes and bladder cancer risk. PLoS One 7, e30665 (2012).

14 Andrew, A. S., Gui, J., Sanderson, A. C., Mason, R. A., Morlock, E. V., Schned, A. R. et al. Bladder cancer SNP panel predicts susceptibility and survival. Hum. Genet. 125, 527-539 (2009).

15 Jung, S. W., Park, N. H., Shin, J. W., Park, B. R., Kim, C. J., Lee, J. E. et al. Prognostic impact of telomere maintenance gene polymorphisms on hepatocellular carcinoma patients with chronic hepatitis B. Hepatology 59, 1912-1920 (2014).
16 Hwang, J. Y., Kim, D. H., Ji, Y. I., Jin Go, M., Heo, L., Jin Kim, Y. et al. Recapitulation of previous genome-wide association studies with two distinct pathophysiological entities of gastric cancer in the Korean population. J. Hum. Genet. 58, 233-235 (2013).

17 Kang, M. Y., Lee, B. B., Ji, Y. I., Jung, E. H., Chun, H. K., Song, S. Y. et al. Association of interindividual differences in p14ARF promoter methylation with single nucleotide polymorphism in primary colorectal cancer. Cancer 112 1699-1707 (2008).

18 Garcia-Magarinos, M., Lopez-de-Ullibarri, I., Cao, R. \& Salas, A. Evaluating the ability of tree-based methods and logistic regression for the detection of SNP-SNP interaction. Ann. Hum. Genet. 73, 360-369 (2009).

19 Huang, J., Lin, A., Narasimhan, B., Quertermous, T., Hsiung, C. A., Ho, L. T. et al. Tree-structured supervised learning and the genetics of hypertension. Proc. Natl Acad. Sci. USA 101, 10529-10534 (2004).

20 Breiman, L. Classification and Regression Trees (Wadsworth International Group, Belmont, CA, USA, 1984).

21 Sole, X., Guino, E., Valls, J., Iniesta, R \& Moreno, V. SNPStats: a web tool for the analysis of association studies. Bioinformatics 22, 1928-1929 (2006).

22 Barrett, J. C., Fry, B., Maller, J. \& Daly, M. J. Haploview: analysis and visualization of LD and haplotype maps. Bioinformatics 21, 263-265 (2005).

23 GTex Consortium. The Genotype-Tissue Expression (GTEx) project. Nat. Genet. 45 , 580-585 (2013).

24 GTex Consortium. Human genomics. The Genotype-Tissue Expression (GTEx) pilot analysis: multitissue gene regulation in humans. Science 348, 648-660 (2015)

25 Pastinen, T. \& Hudson, T. J. Cis-acting regulatory variation in the human genome. Science 306, 647-650 (2004).

$26 \mathrm{Wu}, \mathrm{H}$., Zhang, K., Gong, P., Qiao, F., Wang, L., Cui, H. et al. A nove functional TagSNP Rs7560488 in the DNMT3A1 promoter is associated with susceptibility to gastric cancer by modulating promoter activity. PLoS One 9, e92911 (2014).

27 Weinrich, S. L., Pruzan, R., Ma, L., Ouellette, M., Tesmer, V. M., Holt, S. E. et al. Reconstitution of human telomerase with the template RNA component hTR and the catalytic protein subunit hTRT. Nat. Genet. 17, 498-502 (1997).

28 Liu, Y. Snow, B. E., Hande, M. P. Baerlocher, G., Kickhoefer, V. A., Yeung, D. et al. Telomerase-associated protein TEP1 is not essential for telomerase activity or telomere length maintenance in vivo. Mol. Cell. Biol. 20, 8178-8184 (2000).

29 Bhattacharyya, S., Keirsey, J., Russell, B., Kavecansky, J., Lillard-Wetherell, K., Tahmaseb, K. et al. Telomerase-associated protein 1, HSP90, and topoisomerase Ilalpha associate directly with the BLM helicase in immortalized cells using ALT and modulate its helicase activity using telomeric DNA substrates. J. Biol. Chem. 284, 14966-14977 (2009).

30 Lillard-Wetherell, K., Machwe, A., Langland, G. T., Combs, K. A., Behbehani, G. K. Schonberg, S. A. et al. Association and regulation of the BLM helicase by the telomere proteins TRF1 and TRF2. Hum. Mol. Genet. 13, 1919-1932 (2004).

31 Henson, J. D., Neumann, A. A., Yeager, T. R. \& Reddel, R. R. Alternative lengthening of telomeres in mammalian cells. Oncogene 21, 598-610 (2002).

32 Arinaga, M., Shimizu, S., Gotoh, K., Haruki, N., Takahashi, T., Takahashi, T. et al. Expression of human telomerase subunit genes in primary lung cancer and its clinical significance. Ann. Thorac. Surg. 70, 401-405 discussion 405-406 (2000).

33 Toshikuni, N., Nouso, K., Higashi, T., Nakatsukasa, H., Onishi, T., Kaneyoshi, T. et al. Expression of telomerase-associated protein 1 and telomerase reverse transcriptase in hepatocellular carcinoma. Br. J. Cancer 82, 833-837 (2000).

34 Kameshima, H., Yagihashi, A., Yajima, T., Kobayashi, D., Hirata, K. \& Watanabe, N. Expression of telomerase-associated genes: reflection of telomerase activity in gastric cancer? World J. Surg. 25, 285-289 iv (2001).

35 Ponten, F., Jirstrom, K. \& Uhlen, M. The Human Protein Atlas-a tool for pathology. J. Pathol. 216, 387-393 (2008).

Supplementary Information accompanies the paper on Journal of Human Genetics website (http://www.nature.com/jhg) 